\title{
Age-Specific Differences in the Duration of Prehospital Cardiopulmonary Resuscitation Administered by Emergency Medical Service Providers Necessary to Achieve Favorable Neurological Outcome After Out-of-Hospital Cardiac Arrest
}

\author{
Akira Funada, MD, PhD; Yoshikazu Goto, MD, PhD; Hayato Tada, MD, PhD; Ryota Teramoto, MD; \\ Masaya Shimojima, MD; Kenshi Hayashi, MD, PhD; Masakazu Yamagishi, MD, PhD
}

\begin{abstract}
Background: The appropriate duration of prehospital cardiopulmonary resuscitation (CPR) administered by emergency medical service (EMS) providers for patients with out-of-hospital cardiac arrest (OHCA) necessary to achieve 1-month survival with favorable neurological outcome (Cerebral Performance Category 1 or 2, CPC 1-2) is unclear and could differ by age.

Methods and Results: We analyzed the records of 35,709 adult OHCA patients with return of spontaneous circulation (ROSC) before hospital arrival in a prospectively recorded Japanese registry between 2011 and 2014 . The CPR duration was defined as the time from CPR initiation by EMS providers to prehospital ROSC. The rate of 1-month CPC 1-2 was $21.4 \%(7,650 / 35,709)$. The CPR duration was independently and inversely associated with 1-month CPC 1-2 (adjusted odds ratio, 0.93 per 1-min increment; $95 \%$ confidence interval, 0.93-0.94). The CPR duration increased with age $(P<0.001)$. However, the CPR duration beyond which the proportion of OHCA patients with 1-month CPC 1-2 decreased to $<1 \%$ declined with age: $28 \mathrm{~min}$ for patients aged 18-64 years, $25 \mathrm{~min}$ for $65-74$ years, $23 \mathrm{~min}$ for $75-84$ years, $20 \mathrm{~min}$ for $85-94$ years, and $18 \mathrm{~min}$ for $\geq 95$ years.
\end{abstract}

Conclusions: In patients who achieved prehospital ROSC after OHCA, the duration of CPR administered by EMS providers necessary to achieve 1-month CPC 1-2 varied by age.

Key Words: Aging; Cardiopulmonary resuscitation; Epidemiology; Out-of-hospital cardiac arrest

$\mathbf{W}$ hen emergency medical service (EMS) providers attempt to resuscitate patients in cardiac arrest, their efforts are continued until the patient achieves return of spontaneous circulation (ROSC) or arrival at a hospital where termination of resuscitation (TOR) rules are not applicable. ${ }^{1-3}$ Despite advances in resuscitation knowledge, the appropriate duration of cardiopulmonary resuscitation (CPR) for patients with out-of-hospital cardiac arrest (OHCA) before cessation of CPR remains unclear. Determining the appropriate duration of CPR in the field for OHCA patients is a most important issue. Indeed, the guidelines for CPR do not specify the appropriate duration of CPR before out-ofhospital resuscitation efforts could cease, and information about the duration of resuscitation prior to TOR are lacking for both the prehospital setting and emergency department. ${ }^{1-4}$ Recently, several studies have investigated the relationship between CPR duration and outcome, and in particular according to initial documented rhythm. .-11 $^{5-1}$
Outcomes after OHCA are worse with increasing age, and might be influenced by age-specific differences in response to CPR. ${ }^{12-18}$ Therefore, we hypothesized that the duration of prehospital CPR administered by EMS providers necessary to achieve survival with favorable neurological outcome would differ by age.

In this study, we investigated the relationship between the duration of prehospital CPR administered by EMS providers and survival with favorable neurological outcome after OHCA by age.

\section{Methods}

Study Design and Data Source

In January 2005, the Fire and Disaster Management Agency (FDMA) of Japan launched a prospective, nationwide, population-based, Utstein-style registry that includes all patients with OHCA. ${ }^{13,19}$ The present observational study included adult patients (age $\geq 18$ years) in the registry

Received December 6, 2016; revised manuscript received January 5, 2017; accepted January 11, 2017; released online February 11, 2017 Time for primary review: 21 days

Department of Emergency and Critical Care Medicine, Kanazawa University Hospital, Kanazawa (A.F., Y.G., H.T., R.T., M.S.); Division of Cardiovascular Medicine, Kanazawa University Graduate School of Medical Science, Kanazawa University, Kanazawa (A.F., H.T., R.T., M.S., K.H., M.Y.), Japan

Mailing address: Yoshikazu Goto, MD, PhD, Department of Emergency and Critical Care Medicine, Kanazawa University Hospital, 13-1 Takara-machi, Kanazawa 920-8641, Japan. E-mail: gotoyosh@med.kanazawa-u.ac.jp

ISSN-1346-9843 All rights are reserved to the Japanese Circulation Society. For permissions, please e-mail: cj@j-circ.or.jp 
for whom resuscitation was attempted after OHCA in Japan between January 2011 and December 2014. Some data from this patient cohort from January 2011 to December 2012 have been reported as part of other analyses investigating the relationship between CPR duration and initial documented rhythm. ${ }^{6}$ Cardiac arrest was defined as the cessation of cardiac mechanical activity as confirmed by the absence of signs of circulation. The cause of arrest was presumed to be cardiac unless evidence suggested an external cause, respiratory disease, cerebrovascular disease, malignant tumor, or any other noncardiac cause. Physicians in charge made the determination of the cause of arrest in collaboration with EMS providers.

This study was approved by the Ethics Committee of Kanazawa University with a waiver of informed consent because of the anonymous nature of the data.

\section{The Japanese EMS System}

Japan has approximately 127 million residents in an area of $378,000 \mathrm{~km}^{2}$. Details of the Japanese EMS system have been described previously. ${ }^{13}$ The FDMA of Japan supervises the national EMS system. Each local EMS system is operated by the local fire station. In general, an ambulance crew consists of 3 EMS staff members, including at least 1 emergency lifesaving technician (ELST). ELSTs are allowed to use various resuscitation methods, including

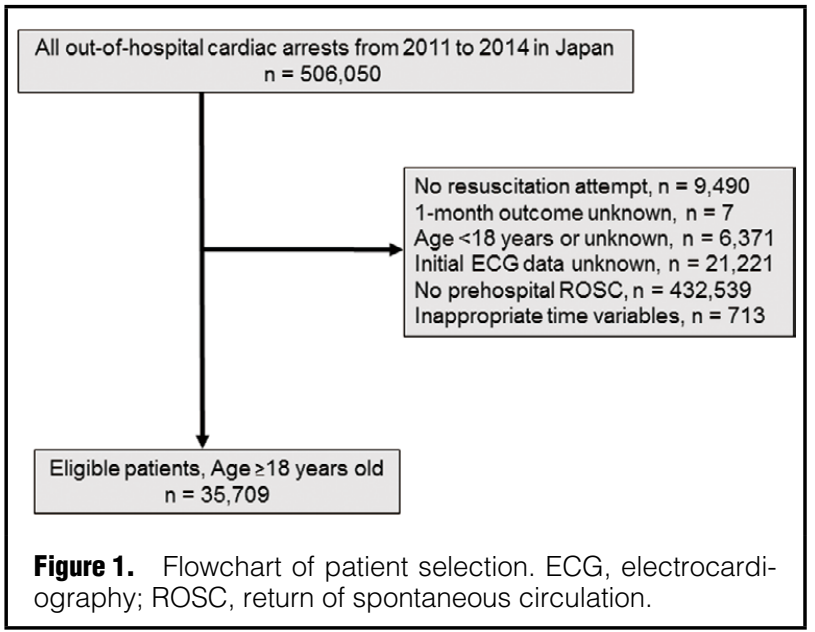

\begin{tabular}{|c|c|c|c|c|c|c|}
\hline Variable & $\begin{array}{c}\text { 18-64 years } \\
(n=9,844)\end{array}$ & $\begin{array}{c}\text { 65-74 years } \\
(n=7,823)\end{array}$ & $\begin{array}{l}75-84 \text { years } \\
(n=10,154)\end{array}$ & $\begin{array}{c}85-94 \text { years } \\
(n=7,007)\end{array}$ & $\begin{array}{l}\geq 95 \text { years } \\
(\mathrm{n}=881)\end{array}$ & $P$ value \\
\hline \multicolumn{7}{|l|}{ Year } \\
\hline 2011 & $2,502(25.4)$ & $1,762(22.5)$ & $2,384(23.5)$ & $1,558(22.2)$ & $207(23.5)$ & $<0.001$ \\
\hline 2012 & $2,516(25.6)$ & $1,935(24.7)$ & $2,463(24.3)$ & $1,685(24.0)$ & $226(25.7)$ & \\
\hline 2013 & $2,412(24.5)$ & $1,979(25.3)$ & $2,560(25.2)$ & $1,848(26.4)$ & $222(25.2)$ & \\
\hline 2014 & $2,414(24.5)$ & $2,147(27.4)$ & $2,747(27.1)$ & $1,916(27.3)$ & $226(25.7)$ & \\
\hline Male sex & $7,131(72.4)$ & $5,548(70.9)$ & $6,345(62.5)$ & $3,236(46.2)$ & $245(27.8)$ & $<0.001$ \\
\hline Witnessed arrest & $7,042(71.5)$ & $5,773(73.8)$ & $7,436(73.2)$ & $5,212(74.4)$ & $670(76.0)$ & $<0.001$ \\
\hline Arrest witnessed by EMS & $1,326(13.5)$ & $1,018(13.0)$ & $1,192(11.7)$ & $793(11.3)$ & $103(11.7)$ & $<0.001$ \\
\hline Presumed cardiac etiology & $6,040(61.4)$ & 4,952 (63.3) & $5,702(56.2)$ & $3,661(52.2)$ & $423(48.0)$ & $<0.001$ \\
\hline Initial shockable rhythm & $4,509(45.8)$ & $2,610(33.4)$ & $1,929(19.0)$ & $750(10.7)$ & $38(4.3)$ & $<0.001$ \\
\hline Bystander CPR & $4,697(47.7)$ & 3,382 (43.2) & $4,496(44.3)$ & $3,504(50.0)$ & $478(54.3)$ & $<0.001$ \\
\hline \multicolumn{7}{|l|}{ EMS response time ( $\mathrm{min})$} \\
\hline Mean \pm SD & $7.2 \pm 3.2$ & $7.3 \pm 3.2$ & $7.4 \pm 3.3$ & $7.4 \pm 3.2$ & $7.3 \pm 3.1$ & \\
\hline Median, IQR & $7(5-9)$ & $7(5-9)$ & $7(5-9)$ & $7(5-9)$ & $7(5-9)$ & $<0.01$ \\
\hline Prehospital AED use & $5,002(50.8)$ & $3,003(38.4)$ & $2,366(23.3)$ & $1,039(14.8)$ & $74(8.4)$ & $<0.001$ \\
\hline Advanced airway management use & $3,418(34.7)$ & $3,275(41.9)$ & $4,823(47.5)$ & $3,519(50.2)$ & $446(50.6)$ & $<0.001$ \\
\hline Adrenaline administration & $3,006(30.5)$ & $2,924(37.4)$ & $4,551(44.8)$ & $3,445(49.2)$ & $429(48.7)$ & $<0.001$ \\
\hline \multicolumn{7}{|l|}{ Outcomes } \\
\hline 1-month survival & $5,003(50.8)$ & 3,201 (40.9) & $2,937(28.9)$ & $1,528(21.8)$ & $127(14.4)$ & $<0.001$ \\
\hline 1-month CPC 1-2 & $3,599(36.6)$ & $1,936(24.7)$ & $1,423(14.0)$ & $655(9.3)$ & $37(4.2)$ & $<0.001$ \\
\hline \multicolumn{7}{|l|}{ CPR duration, overall (min) } \\
\hline Mean \pm SD & $14.3 \pm 9.1$ & $15.3 \pm 9.1$ & $16.5 \pm 9.1$ & $17.2 \pm 9.3$ & $17.4 \pm 9.0$ & \\
\hline Median, IQR & $13(7-20)$ & $14(8-21)$ & $16(10-22)$ & $16(10-23)$ & $17(11-23)$ & $<0.001$ \\
\hline \multicolumn{7}{|l|}{$\begin{array}{l}\text { CPR duration in patients with CPC } 1-2 \\
\text { at } 1 \text { month }(\min )(n=7,650)\end{array}$} \\
\hline Mean \pm SD & $10.3 \pm 6.8$ & $10.3 \pm 7.0$ & $10.7 \pm 7.5$ & $10.4 \pm 7.9$ & $11.4 \pm 9.4$ & \\
\hline Median, IQR & $8(5-13)$ & $8(5-13)$ & $8(5-14)$ & $8(5-14)$ & $9(4-17.5)$ & 0.499 \\
\hline \multicolumn{7}{|l|}{$\begin{array}{l}\text { CPR duration in patients with CPC } 3-5 \\
\text { at } 1 \text { month }(\min )(n=28,059)\end{array}$} \\
\hline Mean \pm SD & $16.7 \pm 9.3$ & $16.9 \pm 9.1$ & $17.5 \pm 8.9$ & $17.9 \pm 9.1$ & $17.7 \pm 8.9$ & \\
\hline Median, IQR & $16(10-22)$ & $16(10-23)$ & $17(11-23)$ & $17(11-23)$ & $17(11-23)$ & $<0.001$ \\
\hline
\end{tabular}

Values are number of patients (\%) unless indicated otherwise. AED, automated external defibrillator; CPC, Cerebral Performance Category scale; CPR, cardiopulmonary resuscitation; EMS, emergency medical services; IQR, interquartile range; SD, standard deviation. 


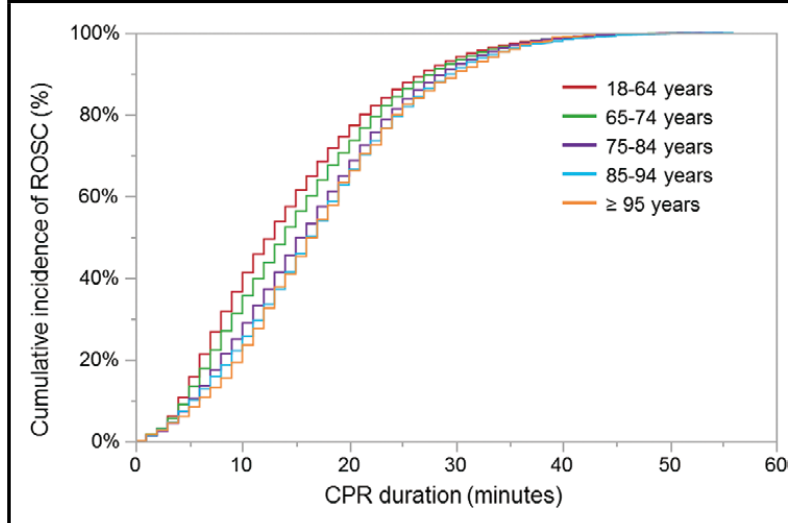

Figure 2. Cumulative incidence of ROSC by age. CPR, cardiopulmonary resuscitation; ROSC, return of spontaneous circulation.

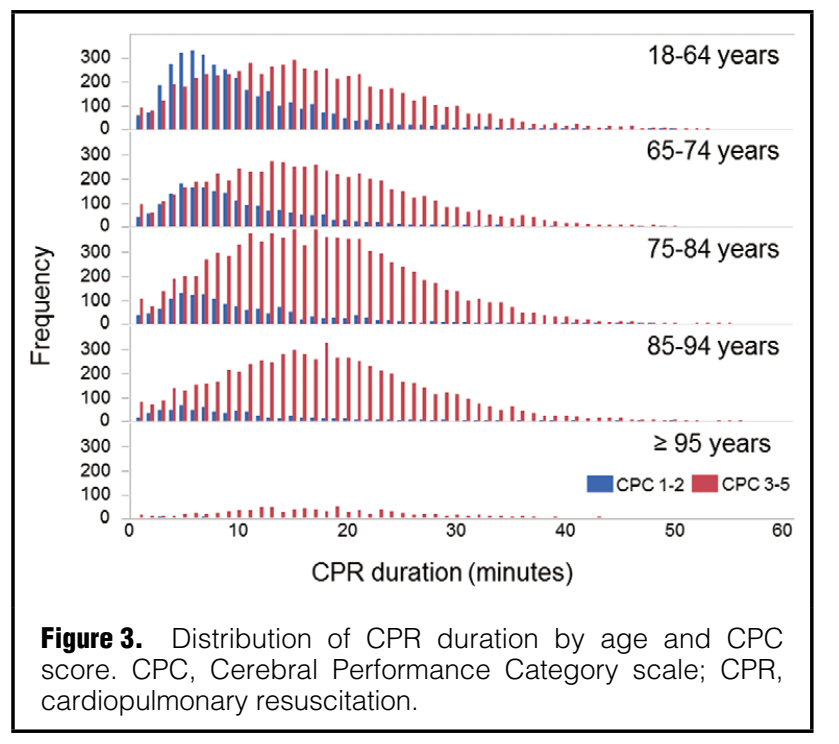

semi-automated external defibrillators (AEDs), insertion of a supraglottic airway device, insertion of a peripheral intravenous line, and administration of Ringer's lactate solution. Since July 2004, only specially trained ELSTs have been permitted to insert a tracheal tube. Since April 2006, they have been permitted to administer intravenous adrenaline in the field under the supervision of an online physician. All EMS providers perform CPR according to the Japanese CPR guidelines. ${ }^{1}$ Because EMS providers in Japan are legally prohibited from TOR in the field, most OHCA patients who receive CPR from EMS providers are transported to hospitals, except in cases of certain death. The duration of on-scene CPR by EMS providers before transport to a hospital was not predetermined.

\section{Data Collection and Quality Control}

The following data were collected prospectively: sex, age, cause of arrest, bystander witness status, bystander CPR with or without AED use, initial cardiac rhythm, bystander category, whether adrenaline was administered, whether advanced airway management techniques were used,

\begin{tabular}{|c|c|}
\hline Variable & $\begin{array}{l}\text { Adjusted OR } \\
(95 \% \mathrm{Cl})\end{array}$ \\
\hline \multicolumn{2}{|l|}{ Year } \\
\hline 2011 & Ref. \\
\hline 2012 & $0.95(0.86-1.04)$ \\
\hline 2013 & $1.02(0.93-1.12)$ \\
\hline 2014 & $0.98(0.90-1.07)$ \\
\hline \multicolumn{2}{|l|}{ Age group } \\
\hline $18-64$ years & Ref. \\
\hline $65-74$ years & $0.66(0.61-0.71)$ \\
\hline $75-84$ years & $0.45(0.42-0.49)$ \\
\hline $85-94$ years & $0.36(0.32-0.40)$ \\
\hline$\geq 95$ years & $0.18(0.12-0.25)$ \\
\hline Male sex & $1.08(1.00-1.16)$ \\
\hline Witnessed arrest & $1.72(1.58-1.87)$ \\
\hline Arrest witnessed by EMS & $2.42(2.19-2.66)$ \\
\hline Presumed cardiac etiology & $2.14(1.97-2.32)$ \\
\hline Initial shockable rhythm & $2.89(2.55-3.29)$ \\
\hline Bystander CPR & $1.03(0.96-1.11)$ \\
\hline EMS response time (per 1-min) & $0.96(0.95-0.97)$ \\
\hline Prehospital AED use & $1.56(1.37-1.77)$ \\
\hline Advanced airway management use & $0.61(0.56-0.66)$ \\
\hline Adrenaline administration & $0.26(0.24-0.29)$ \\
\hline CPR duration (per 1-min) & $0.93(0.93-0.94)$ \\
\hline
\end{tabular}

$\mathrm{Cl}$, confidence interval; OHCA, out-of-hospital cardiac arrest; OR, odds ratio. Other abbreviations as in Table 1.

whether ROSC was achieved before arrival at the hospital, time of the emergency call, time of vehicle arrival at the scene, time of CPR initiation by EMS providers, time of ROSC, time of vehicle arrival at the hospital, time of adrenaline administration, time of shock delivery by EMS providers, 1-month survival, and neurological outcome at 1 month after cardiac arrest. EMS response time was calculated as the time from the emergency call to the time of vehicle arrival at the scene. CPR duration was defined as the time from CPR initiation by EMS providers to prehospital ROSC. ${ }^{6,9}$ Neurological outcome was defined using the Cerebral Performance Category (CPC) scale: category 1, good cerebral performance; category 2, moderate cerebral disability; category 3 , severe cerebral disability; category 4 , coma or vegetative state; and category 5 , death. ${ }^{19}$ The physician in charge determined the CPC category.

\section{Outcome}

The primary study outcome was 1-month survival with favorable neurological outcome, defined as a CPC score of 1 or 2 (CPC 1-2).

\section{Statistical Analysis}

Continuous variables are expressed as medians (interquartile range) or means and standard deviations. Continuous variables were compared with the Kruskal-Wallis test. Categorical variables are expressed as counts (\%), and differences between groups were compared using the chisquare test. We classified the following 2 covariates into several categories: age $(18-64,65-74,75-84,85-94$, and $\geq 95$ years) and CPR duration $(0-5,6-10,11-15,16-20$, $21-25,26-30,31-35$, and $\geq 36 \mathrm{~min})$. The cumulative inci- 
Table 3. Adjusted ORs and 95\% Cls of CPR Duration and Other Prehospital Factors for 1-Month CPC 1-2 After OHCA in Multivariate Logistic Regression Model by Age

\section{Variable}

(A) CPR duration as continuous variable

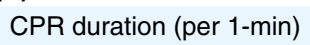

Male sex

Witnessed arrest

Arrest witnessed by EMS

Presumed cardiac etiology

Initial shockable rhythm

Bystander CPR

EMS response time (per 1-min)

Prehospital AED use

Advanced airway management use

Adrenaline administration

(B) CPR duration as categorical variable

CPR duration (min)

$\begin{array}{lc}0-5 & 5.56(3.45-9.25) \\ 6-10 & 4.54(2.84-7.49) \\ 11-15 & 2.65(1.66-4.38) \\ 16-20 & 2.25(1.40-3.74) \\ 21-25 & 1.30(0.79-2.21) \\ 26-30 & 1.19(0.69-2.07) \\ 31-35 & 1.01(0.54-1.89) \\ \geq 36 & \text { Ref. }\end{array}$

Year

2011

2012

2013

2014

Male sex

Witnessed arrest

Arrest witnessed by EMS

Presumed cardiac etiology

Initial shockable rhythm

Bystander CPR

EMS response time (per 1-min)

Prehospital AED use

Advanced airway management use

Adrenaline administration

$\begin{gathered}18-64 \text { years } \\ (\mathbf{n}=9,844)\end{gathered}$
$0.94(0.93-0.95)$
Ref.
$0.87(0.75-1.01)$
$1.00(0.86-1.17)$
$1.00(0.86-1.17)$
$1.15(1.01-1.31)$
$1.56(1.36-1.78)$
$2.21(1.86-2.63)$
$2.85(2.46-3.30)$
$2.72(2.24-3.32)$
$1.18(1.05-1.33)$
$0.96(0.94-0.98)$
$1.98(1.61-2.44)$
$0.63(0.56-0.72)$
$0.30(0.25-0.35)$

Ref.

$0.87(0.75-1.01)$

$1.00(0.86-1.17)$

$1.00(0.86-1.16)$

$1.15(1.01-1.31)$

$1.55(1.35-1.77)$

$2.20(1.85-2.62)$

2.87 (2.48-3.32)

2.73 (2.24-3.32)

$1.18(1.05-1.33)$

$0.96(0.95-0.98)$

$1.97(1.60-2.43)$

$0.65(0.57-0.74)$

$0.30(0.26-0.36)$

\section{5-74 years}

$(\mathrm{n}=7,823)$

$0.94(0.93-0.95)$

Ref.

0.83 (0.69-0.99)

$0.97(0.81-1.16)$

$0.88(0.74-1.06)$

$1.13(0.98-1.32)$

$1.84(1.55-2.18)$

2.46 (2.03-2.98)

$2.38(2.01-2.82)$

$2.90(2.25-3.75)$

$1.12(0.97-1.28)$

$0.96(0.94-0.98)$

$1.40(1.07-1.81)$

$0.61(0.53-0.71)$

$0.26(0.21-0.31)$

\section{5-84 years}

$(n=10,154)$

$0.93(0.92-0.94)$

Ref.

$1.14(0.95-1.38)$

$1.08(0.89-1.30)$

$1.06(0.88-1.28)$

$0.88(0.77-1.01)$

$1.76(1.47-2.11)$

2.74 (2.28-3.30)

$1.81(1.55-2.12)$

$3.43(2.58-4.60)$

$0.87(0.75-1.00)$

$0.97(0.94-0.99)$

$1.21(0.90-1.60)$

$0.57(0.49-0.67)$

$0.24(0.19-0.30)$
85-94 years

$(n=7,007)$

$0.92(0.91-0.93)$

Ref.

$1.08(0.84-1.40)$

1.09 (0.84-1.41)

$1.00(0.77-1.29)$

$1.00(0.83-1.19)$

$1.49(1.16-1.91)$

$2.40(1.87-3.07)$

$1.23(1.01-1.50)$

$2.66(1.77-4.05)$

$0.81(0.66-0.98)$

$0.98(0.95-1.01)$

$1.24(0.82-1.85)$

$0.57(0.45-0.72)$

$0.20(0.14-0.28)$

Abbreviations as in Tables 1,2.

dence of ROSC was calculated using the Kaplan-Meier method and compared using the log-rank test. Outcomes by age group were compared using the Cochran-Armitage trend test. Multivariate logistic regression models that included potential confounders based on biological plausibility and previous studies were used to identify factors associated with 1-month CPC 1-2; odds ratios (ORs) and their $95 \%$ confidence intervals (CIs) were calculated for all patients, as well as for each age group except for patients aged $\geq 95$ years because of the limited number of 1-month CPC 1-2 events. The dynamic proportion of 1-month CPC
1-2 was calculated for all patients and each age group, using the following formula: dynamic proportion of a 1 -month CPC $1-2(Y)[\%]=\{[$ survivors with CPC $1-2$ at 1 month after OHCA, for all patients or each age group)$N x] \times 100\} /($ all patients or each age group with OHCA), where $N x$ is the number of all patients or each age group who received CPR for 0 to $x$ min and survived with CPC 1-2 for 1 month after OHCA. ${ }^{6,9}$ The cumulative proportion of 1-month CPC 1-2 was also calculated for all patients and each age groups, using the following formula: cumulative proportion of 1-month CPC 1-2 (Y) 
$[\%]=(N x \times 100) /($ survivors with CPC $1-2$ at 1 month after OHCA, for all patients or each age group), where $N x$ is the number of all patients or each age group who received CPR for 0 to $x$ min and survived with CPC 1-2 for 1 month after OHCA. ${ }^{6,9}$ On the basis of medical futility $(<1 \%$ chance of 1 -month CPC $1-2),{ }^{20}$ we determined 2 categories of CPR duration: that beyond which the dynamic proportion of 1-month CPC 1-2 decreased to $<1 \%$, and that necessary to achieve a $>99 \%$ cumulative proportion of OHCA patients with 1-month CPC 1-2.5-11

All statistical analyses were performed using JMP Pro software, version 12.2.0 (SAS Institute, Cary, NC, USA).

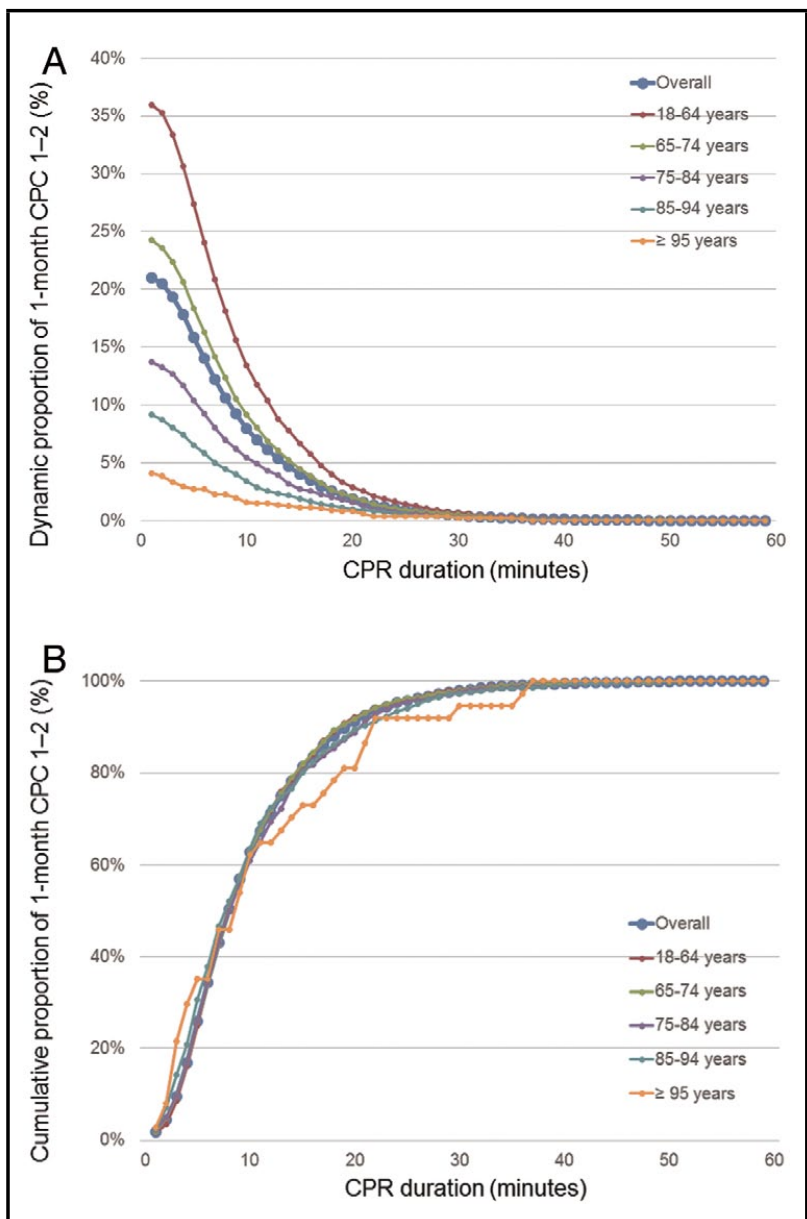

Figure 4. (A) Dynamic proportion of 1-month CPC 1-2 by age and (B) Cumulative proportion of 1-month CPC 1-2 by age. Abbreviations as in Figure 3.
All tests were 2-tailed, and $\mathrm{P}<0.05$ was considered statistically significant.

\section{Results}

\section{Patients' and EMS Characteristics}

During the 4-year study period, 506,050 OHCA events were documented. Of 496,560 OHCAs with attempted resuscitation, the $35,709(7.2 \%)$ patients who were treated by EMS providers and achieved prehospital ROSC were eligible for our analysis (Figure 1). Patients' and EMS characteristics are shown in Table 1. The proportion of males and initial documented shockable rhythm decreased with age. The proportion of prehospital AED administration decreased with age, and the use of advanced airway management increased with age.

\section{Outcomes}

Overall, the rate of 1-month CPC 1-2 after OHCA was $21.4 \%(7,650 / 35,709)$. When categorized by age group, the rate of 1 -month CPC $1-2$ was $36.6 \%(3,599 / 9,844)$ for patients aged $18-64$ years, $24.7 \%(1,936 / 7,823)$ for 65-74 years, $14.0 \%(1,423 / 10,154)$ for $75-84$ years, $9.3 \%$ $(655 / 7,007)$ for $85-94$ years, and $4.2 \%(37 / 881)$ for $\geq 95$ years $(\mathrm{P}$ for trend $<0.001)$ (Table $\mathbf{1})$.

\section{Prehospital CPR Duration}

Prehospital CPR duration increased with age (Table 1). The cumulative incidence of prehospital ROSC by age group is shown in Figure 2. Younger age was associated with earlier ROSC (log-rank $\mathrm{P}<0.001)$. The distribution of CPR duration was highly variable, skewed towards shorter duration in younger patients and shifted to longer duration with age (Figure 3). When CPC score was stratified into 2 groups, CPC 1-2 and CPC 3-5, CPR duration did not differ among the 5 age groups in the CPC 1-2 group, but CPR duration increased with age in the CPC 3-5 group (Table 1, Figure 3). CPR duration was independently and inversely associated with 1-month CPC $1-2$ in all patients (adjusted OR, 0.93 per 1-min increase; 95\% CI, 0.93-0.94) (Table 2). Table 3 shows the adjusted ORs of CPR duration for 1-month CPC 1-2 by age group. CPR duration (as a continuous variable) was independently and inversely associated with 1-month CPC 1-2 in all age groups. When CPR duration was sorted as a categorical variable, it was independently associated with improved 1-month CPC $1-2$ : $\leq 20 \mathrm{~min}$ for patients aged $18-64$ years, $\leq 15 \mathrm{~min}$ for patients aged $65-74$ and $75-84$ years, and $\leq 10 \mathrm{~min}$ for patients aged $85-94$ years.

\section{Dynamic Proportion of 1-Month CPC 1-2}

The CPR duration beyond which the dynamic proportion of 1-month CPC $1-2$ decreased to $<1 \%$ was 25 min for all

\begin{tabular}{|c|c|c|c|c|c|c|}
\hline & $\begin{array}{c}\text { All patients } \\
(n=35,709)\end{array}$ & $\begin{array}{c}18-64 \text { years } \\
(n=9,844)\end{array}$ & $\begin{array}{c}65-74 \text { years } \\
(n=7,823)\end{array}$ & $\begin{array}{c}75-84 \text { years } \\
(n=10,154)\end{array}$ & $\begin{array}{c}85-94 \text { years } \\
(n=7,007)\end{array}$ & $\begin{array}{c}\geq 95 \text { years } \\
(n=881)\end{array}$ \\
\hline \multicolumn{7}{|c|}{ Cumulative proportion of 1-month CPC 1-2 } \\
\hline$>90 \%$ & $\leq 20 \min$ & $\leq 19 \min$ & $\leq 19 \min$ & $\leq 21 \mathrm{~min}$ & $\leq 21 \mathrm{~min}$ & $\leq 22 \min$ \\
\hline$>99 \%$ & $\leq 35 \mathrm{~min}$ & $\leq 34 \mathrm{~min}$ & $\leq 34 \mathrm{~min}$ & $\leq 37 \mathrm{~min}$ & $\leq 39 \min$ & $37 \mathrm{~min}$ \\
\hline $100 \%$ & $52 \mathrm{~min}$ & $50 \mathrm{~min}$ & $52 \mathrm{~min}$ & $48 \mathrm{~min}$ & $50 \mathrm{~min}$ & $37 \mathrm{~min}$ \\
\hline
\end{tabular}

Abbreviations as in Tables 1,2. 
patients. When stratified by age group, the duration was $28 \mathrm{~min}$ for patients aged 18-64 years, $25 \mathrm{~min}$ for $65-74$ years, $23 \mathrm{~min}$ for $75-84$ years, $20 \mathrm{~min}$ for $85-94$ years, and $18 \mathrm{~min}$ for $\geq 95$ years (Figure 4A).

\section{Cumulative Proportion of 1-Month CPC 1-2}

The CPR duration to achieve $>99 \%$ cumulative proportion of 1-month CPC 1-2 was $35 \mathrm{~min}$ for all patients, $34 \mathrm{~min}$ for patients aged 18-64 years, $34 \mathrm{~min}$ for $65-74$ years, $37 \mathrm{~min}$ for 75-84 years, $39 \mathrm{~min}$ for $85-94$ years, and $37 \mathrm{~min}$ for $\geq 95$ years (Figure 4B, Table 4).

\section{Discussion}

This observational study of patients with prehospital ROSC from a Japanese OHCA registry demonstrated that for prehospital CPR administered by EMS providers, the duration necessary to achieve 1-month CPC 1-2 differed by age. In the present study, the CPR duration was independently and inversely associated with 1-month CPC 1-2 after adjusting for prehospital variables. The rate of 1-month CPC 1-2 decreased with age. CPR duration increased with age (Table 1). Furthermore, CPR duration (as a categorical variable) associated with beneficial effects shortened with age (Table 3). These differences might be influenced by differences in the etiology of OHCA between age groups, because the proportion of patients with presumed cardiac etiology decreased with age (Table 1). Furthermore, the proportion with initial shockable rhythm and prehospital AED administration decreased with age.

An objective criterion for medical futility has previously been defined as $<1 \%$ chance of favorable outcome for interventions and drug therapy, and this remains the basis for current resuscitation research.,20 However, the relationship between medical futility and ethical responsibility remains controversial. ${ }^{21}$ Thus, we determined the CPR duration beyond which the dynamic proportion of 1-month CPC $1-2$ decreased to $<1 \%$, and found that this duration shortened with age, which suggests that compared with elderly patients, younger patients have a greater ability to achieve 1-month CPC 1-2 after longer CPR duration. Furthermore, the dynamic proportion of 1-month CPC 1-2 was nearly identical in all age groups, at $<1 \%$, for CPR duration of $\approx 30 \mathrm{~min}$. This suggests that younger patients were more responsive to early-phase CPR than elderly patients. These age-specific differences in response to CPR might be associated with the observed differences in the rates of 1-month CPC 1-2 among age groups, and could provide useful information for EMS providers regarding whether to continue CPR during transportation to hospital and for physicians on whether to terminate or withhold CPR immediately after patient arrival in the emergency department based on age. To date, the USA and other Western countries have implemented a universal TOR rule defined by 3 mandatory criteria (EMS-unwitnessed arrest, no prehospital ROSC, and no shock delivery), ${ }^{4}$ but age is not accounted for. We suggest that age-specific differences in response to CPR might be taken into consideration to reduce futile resuscitation, given limited medical resources, and to improve the present TOR rules. However, we also found that the CPR duration was similar across age groups in patients with 1-month CPC 1-2, although CPR duration increased with age in patients with 1-month CPC 3-5. Therefore, age alone might have less effect on CPR duration in terms of achieving 1-month CPC 1-2 than other established factors such as cardiac etiology, witnessed arrest, initial shockable rhythm, and bystander CPR. ${ }^{6-11}$

On the other hand, the CPR duration necessary to achieve $>99 \%$ cumulative proportion of OHCA patients with 1-month CPC 1-2 increased with age (Figure 4B, Table 4). Elderly patients require longer CPR duration to achieve ROSC, and their prognosis is generally poor. However, a substantial proportion had meaningful recovery after prolonged CPR. ${ }^{22}$ In the present study, the ratios of remaining patients with 1-month CPC 1-2 to all patients or to each age group with 1-month CPC 1-2 following a CPR duration beyond which the dynamic proportion of 1 -month CPC 1-2 decreased to $<1 \%$ were $4.2 \%(319 / 7,650)$ for all patients, $2.4 \%(88 / 3,599)$ for patients aged $18-64$ years, 3.7\% $(72 / 1,936)$ for $65-74$ years, $6.2 \%(88 / 1,423)$ for 75-84 years, $10.5 \%(69 / 655)$ for $85-94$ years, and $21.6 \%$ $(8 / 37)$ for $\geq 95$ years. Therefore, longer CPR duration than that beyond which the dynamic proportion of 1-month CPC 1-2 decreased to $<1 \%$ might be more effective in elderly patients than in younger patients. However, the effect of CPR lasting longer than this duration would probably be very small because in this study there were few remaining patients with 1 -month CPC 1-2. In patients with in-hospital cardiac arrest without ROSC, younger age is associated with longer CPR duration, probably because of the perception of a higher possibility of survival. ${ }^{23}$ In the present study, contrary to our expectation that a selffulfilling prophecy might result in shortening the CPR duration in elderly patients, elderly patients had longer CPR duration than younger patients. ${ }^{15,24}$ Conceivably, because we investigated only patients with prehospital ROSC, elderly patients who needed longer CPR duration to achieve ROSC were included.

In many EMS systems in the USA and other Western countries, patients with OHCA are declared dead at the scene after a predetermined CPR duration, often $30 \mathrm{~min} .{ }^{25-27}$ In the present study, the CPR duration beyond which the dynamic proportion of 1-month CPC 1-2 decreased to $<1 \%$ was $<30 \mathrm{~min}$, but the CPR duration necessary to achieve $>99 \%$ cumulative proportion of 1 -month CPC $1-2$ was $>30 \mathrm{~min}$. These results were consistent with those from other recent reports. ${ }^{\mathbf{6 8 , 1 0 , 1 1}}$ Therefore, for CPR to achieve the maximum favorable outcomes, CPR duration $>30 \mathrm{~min}$ might be required. However, we investigated only patients with prehospital ROSC to identify the prehospital duration of CPR needed to achieve 1-month CPC 1-2 when administered by EMS providers. When including patients without prehospital ROSC, it seems that the CPR duration beyond which the dynamic proportion of 1-month CPC $1-2$ decreases to $<1 \%$ might decline and the CPR duration necessary to achieve $>99 \%$ cumulative proportion of 1-month CPC 1-2 might increase. This hypothesis needs further prospective investigation.

Several studies examining the effect of CPR duration after OHCA by initial documented rhythm have reported that initial non-shockable rhythm is associated with a shorter CPR duration beyond which the proportion of outcome decreased to $<1 \%$ and is also associated with a longer CPR duration needed to achieve $>99 \%$ cumulative proportion of outcomes. ${ }^{\mathbf{6 8 , 1 1}}$ In these studies, patients with non-shockable rhythms were older than those with shockable rhythms. In the present study, the proportion of nonshockable rhythm increased with age. On a physiological level, it is conceivable that older age might make nonshockable rhythms more common, so it is possible that it 
is older age, not non-shockable rhythm, that is the fundamental determinant of CPR duration. However, at the scene, stratification by initial documented rhythm might be more feasible because initial shockable rhythm is a strong predictor of favorable outcome and the CPR strategy differs by initial documented rhythm. ${ }^{28}$

In this study, we could not investigate the duration of bystander CPR in detail because both the time of initiation and duration of bystander CPR was unreliable compared with CPR administered by EMS providers. Unfortunately, we had no data regarding the quality of bystander CPR, namely, whether CPR performed by bystanders was appropriate or not. Furthermore, the effectiveness of bystander CPR is influenced by factors such as bystander characteristics (e.g., family member, non-family member, trained layperson, or elderly person with physical limitations), type of bystander CPR (e.g., conventional, chest compressions only, or ventilation only), and self-fulfilling prophecy related to advanced age. ${ }^{\mathbf{1 2 , 1 5 , 2 4}}$ Indeed, bystander CPR was not associated with 1-month CPC 1-2 in all patients. When stratified by age, bystander CPR was positively associated with 1-month CPC $1-2$ in patients aged 18-64 years and negatively associated with 1-month CPC 1-2 in patients aged 85-94 years (Table 3). Therefore, to investigate CPR duration, including the duration of bystander CPR in the field before EMS provider arrival, further prospective studies that assess the quality of bystander CPR are needed.

\section{Study Limitations}

First, we could not analyze most of the OHCA patients who were transported to hospitals without prehospital ROSC because of the lack of data including in-hospital CPR duration. Therefore, further prospective study including the duration of in-hospital CPR may be required to strengthen the present results. Second, we did not have sufficient data to permit further risk adjustment (e.g., comorbid disease, location of cardiac arrest, quality of EMS providers, CPR quality, and in-hospital medication). This inability is attributable to the study design, which was a retrospective record review. Third, further validation studies are needed to interpret the results of the present study in other countries or EMS systems, depending on whether TOR is applicable. Finally, as with all epidemiological studies, the integrity, validity, and ascertainment bias of the data are potential limitations. The large sample size, uniform Utstein-style procedures for collecting data on cardiac arrest, and a population-based study design covering all known OHCAs in Japan were intended to minimize these potential sources of bias.

\section{Conclusions}

In patients who achieved prehospital ROSC after OHCA, the duration of CPR administered by EMS providers necessary to achieve 1-month CPC 1-2 varied by age. Age-specific differences in response to CPR could be helpful for making decisions on terminating or withholding CPR based on age.

\section{Acknowledgments}

We thank all the EMS providers and participating physicians in Japan and the FDMA for their generous cooperation in establishing and maintaining the database.

\section{Funding Source}

This work was supported by JSPS KAKENHI Grant No. JP16K19171.

\section{Conflicts of Interest}

None.

\section{References}

1. Japan Resuscitation Council. 2010 Japanese guidelines for emergency care and cardiopulmonary resuscitation. Tokyo: Health Shuppansha, 2011 (in Japanese).

2. American Heart Association. 2010 American Heart Association guidelines for cardiopulmonary resuscitation and emergency cardiovascular care. Circulation 2010; 122: S639-S946.

3. Nolan JP, Soar J, Zideman DA, Biarent D, Bossaert LL, Deakin $\mathrm{C}$, et al. European Resuscitation Council guidelines for resuscitation 2010 section 1: Executive summary. Resuscitation 2010; 81: $1219-1276$.

4. Morrison LJ, Visentin LM, Kiss A, Theriault R, Eby D, Vermeulen $\mathrm{M}$, et al. Validation of a rule for termination of resuscitation in out-of-hospital cardiac arrest. N Engl J Med 2006; 355: 478-487.

5. Reynolds JC, Frisch A, Rittenberger JC, Callaway CW. Duration of resuscitation efforts and functional outcome after out-of-hospital cardiac arrest: When should we change to novel therapies? Circulation 2013; 128: 2488-2494.

6. Goto Y, Funada A, Goto Y. Relationship between the duration of cardiopulmonary resuscitation and favorable neurological outcomes after out-of-hospital cardiac arrest: A prospective, nationwide, population-based cohort study. J Am Heart Assoc 2016; 4: e002819.

7. Nagao K, Nonogi H, Yonemoto N, Gaieski DF, Ito N, Takayama $\mathrm{M}$, et al. Duration of prehospital resuscitation efforts after out-of-hospital cardiac arrest. Circulation 2016; 133: 1386-1396.

8. Grunau B, Reynolds JC, Scheuermeyer FX, Stenstrom R, Pennington S, Cheung C, et al. Comparing the prognosis of those with initial shockable and non-shockable rhythms with increasing durations of CPR: Informing minimum durations of resuscitation. Resuscitation 2016; 101: 50-56.

9. Goto Y, Funada A, Goto Y. Duration of prehospital cardiopulmonary resuscitation and favorable neurological outcomes for pediatric out-of-hospital cardiac arrests: A nationwide, population-based cohort study. Circulation 2016; 134: 2046-2059.

10. Nehme Z, Andrew E, Bernard S, Smith K. Impact of cardiopulmonary resuscitation duration on survival from paramedic witnessed out-of-hospital cardiac arrests: An observational study. Resuscitation 2016; 100: 25-31.

11. Reynolds JC, Grunau BE, Rittenberger JC, Sawyer KN, Kurz $\mathrm{MC}$, Callaway CW. The association between duration of resuscitation and favorable outcome after out-of-hospital cardiac arrest: Implications for prolonging or terminating resuscitation. Circulation 2016; 134: 2084-2094.

12. Funada A, Goto Y, Maeda T, Teramoto R, Hayashi K, Yamagishi M. Improved survival with favorable neurological outcome in elderly individuals with out-of-hospital cardiac arrest in Japan: A nationwide observational cohort study. Circ J 2016; 80: $1153-1162$.

13. Kitamura T, Iwami T, Kawamura T, Nitta M, Nagao K, Nonogi H, et al. Nationwide improvements in survival from out-of-hospital cardiac arrest in Japan. Circulation 2012; 126: 2834-2843.

14. Fukuda T, Ohashi-Fukuda N, Matsubara T, Doi K, Kitsuta Y, Nakajima S, et al. Trends in outcomes for out-of-hospital cardiac arrest by age in Japan: An observational study. Medicine (Baltimore) 2015; 94: e2049.

15. Andersen LW, Bivens MJ, Giberson T, Giberson B, Mottley JL, Gautam S, et al. The relationship between age and outcome in out-of-hospital cardiac arrest patients. Resuscitation 2015; 94: $49-54$.

16. Wissenberg M, Folke F, Hansen CM, Lippert FK, Kragholm K, Risgaard B, et al. Survival after out-of-hospital cardiac arrest in relation to age and early identification of patients with minimal chance of long-term survival. Circulation 2015; 131: 1536-1545.

17. Funada A, Goto Y, Tada H, Teramoto R, Shimojima M, Hayashi K, et al. Age-specific differences in prognostic significance of rhythm conversion from initial non-shockable to shockable rhythm and subsequent shock delivery in out-of-hospital cardiac arrest. Resuscitation 2016; 108: 61-67.

18. Funada A, Goto Y, Maeda T, Tada H, Teramoto R, Tanaka Y, 
et al. Prehospital predictors of neurological outcomes in out-ofhospital cardiac arrest patients aged 95 years and older: A nationwide population-based observational study. J Cardiol 2017; 69: $340-344$

19. Jacobs I, Nadkarni V, Bahr J, Berg RA, Billi JE, Bossaert L, et al. Cardiac arrest and cardiopulmonary resuscitation outcome reports: Update and simplification of the Utstein templates for resuscitation registries: A statement for healthcare professionals from a task force of the International Liaison Committee on Resuscitation (American Heart Association, European Resuscitation Council, Australian Resuscitation Council, New Zealand Resuscitation Council, Heart and Stroke Foundation of Canada, InterAmerican Heart Foundation, Resuscitation Councils of Southern Africa). Circulation 2004; 110: 3385-3397.

20. Schneiderman LJ, Jecker NS, Jonsen AR. Medical futility: Its meaning and ethical implications (Review). Ann Intern Med 1990; 112: 949-954.

21. Goto Y, Maeda T, Goto YN. Termination-of-resuscitation rule for emergency department physicians treating out-of-hospital cardiac arrest patients: An observational cohort study. Crit Care 2013; 17: R235.

22. Rajan S, Folke F, Kragholm K, Hansen CM, Granger CB, Hansen SM, et al. Prolonged cardiopulmonary resuscitation and outcomes after out-of-hospital cardiac arrest. Resuscitation 2016;
105: $45-51$.

23. Khan AM, Kirkpatrick JN, Yang L, Groeneveld PW, Nadkarni VM, Merchant RM, et al. Age, sex, and hospital factors are associated with the duration of cardiopulmonary resuscitation in hospitalized patients who do not experience sustained return of spontaneous circulation. J Am Heart Assoc 2014; 3: e001044.

24. Nehme Z, Andrew E, Bernard S, Smith K. The impact of partial resuscitation attempts on the reported outcomes of out-ofhospital cardiac arrest in Victoria, Australia: Implications for Utstein-style outcome reports. Resuscitation 2014; 85: 1185-1191.

25. Atkins DL. Doing the same thing over and over, yet expecting different results. Circulation 2013; 128: 2465-2467.

26. Bailey ED, Wydro GC, Cone DC. Termination of resuscitation in the prehospital setting for adult patients suffering nontraumatic cardiac arrest: National Association of EMS Physicians Standards and Clinical Practice Committee. Prehosp Emerg Care 2000; 4: 190-195.

27. Cone DC, Bailey ED, Spackman AB. The safety of a field termination-of-resuscitation protocol. Prehosp Emerg Care 2005; 9: $276-281$

28. Sasson C, Rogers MA, Dahl J, Kellermann AL. Predictors of survival from out-of-hospital cardiac arrest: A systematic review and meta-analysis. Circ Cardiovasc Qual Outcomes 2010; 3: 63-81. 